\title{
CARACTERIZAÇÃO DO PERFIL SOCIODEMOGRÁFICO, DAS CONDIÇÕES DE SAÚDE E DAS CONDIÇÕES SOCIAIS DE IDOSOS OCTOGENÁRIOS
}

\section{Matheus Santos Gomes Jorge}

Fisioterapeuta, Pós-graduando do Curso de Especialização em Fisioterapia Traumato-ortopédica e mestrando do Programa de Pós-graduação em Envelhecimento Humano, Universidade de Passo Fundo (UPF), Passo Fundo (RS), Brasil Brasil.

\section{E-mail: mathjorge5@gmail.com}

\section{Willian Guerra de Lima}

Graduando do Curso de Fisioterapia, Faculdade de Educação Física e Fisioterapia, Universidade de Passo Fundo (UPF); Bolsista PIBIC/CNPq. Brasil.

\section{Patrícia Rodigheri Vieira}

Graduanda do Curso de Fisioterapia, Faculdade de Educação Física e Fisioterapia, Universidade de Passo Fundo (UPF); Bolsista PIBIC/CNPq. Brasil.

\section{Sabrina Casarin Vogelmann}

Graduanda do Curso de Fisioterapia, Faculdade de Educação Física e Fisioterapia, Universidade de Passo Fundo (UPF), Brasil.

\section{Rafaela Simon Myra}

Fisioterapeuta pela Universidade de Passo Fundo (UPF); Mestranda do Programa de Pós-graduação em Fisioterapia, Universidade do Estado de Santa Catarina, Florianópolis (SC), Brasil.

\section{Lia Mara Wibelinger}

Doutora em Gerontologia Biomédica pela Pontifícia Universidade Católica (PUCRS); Docente do Curso de Fisioterapia e do Programa de Pós-graduação em Envelhecimento Humano, Faculdade de Educação Física e Fisioterapia, Universidade de Passo Fundo (UPF), Brasil.
RESUMO: O objetivo deste estudo foi caracterizar o perfil sociodemográfico, as condições de saúde e as condições sociais de idosos octogenários. Estudo quantitativo, descritivo de corte transversal, que avaliou o perfil sociodemográfico (12 itens), as condições de saúde (15 itens) e as condições sociais ( 04 itens) de 58 idosos octogenários, por meio de um questionário estruturado, totalizando 31 itens avaliados. Os resultados demonstraram que a amostra era predominantemente feminina (68,96\%), viúva (62,06\%), com 80-89 anos (79,31\%), com escolaridade básica $(89,63 \%)$ e aposentada $(75,86 \%)$. Possuíam casa própria $(65,51 \%)$, tinham filhos e/ou netos $(89,65 \%)$, eram católicos $(84,48 \%)$, tinham plano de saúde $(58,62 \%)$, apresentavam alterações oculares $(72,41 \%)$ e faziam uso de dispositivos para correção $(72,41 \%)$. A maioria apresentava hipertensão arterial sistêmica (55,17\%), fazia uso de hipotensores $(55,17 \%)$, realizava consultas médicas regulares $(63,79 \%)$, já realizou fisioterapia $(53,44 \%)$, praticava atividade física $(65,11 \%)$ e classificou sua saúde como regular ou boa $(43,10 \%)$. Em suma, o perfil sociodemográfico, as condições de saúde e as condições sociais dos idosos descritas neste estudo podem contribuir para o conhecimento sobre os octogenários, o que torna isto uma questão de saúde pública em virtude do progressivo crescimento populacional destes indivíduos.

PALAVRAS-CHAVE: Idoso de 80 anos ou mais; Idoso; Longevidade; Perfil de saúde; Condições sociais.

\section{SOCIAL AND DEMOGRAPHIC PROFILE, HEALTH CONDITIONS AND SOCIAL CONDITIONS OF OVER- EIGHTY ELDERLY PEOPLE}

\begin{abstract}
The social and demographic profile, health and social conditions of elderly people aged 80 or more are analyzed in current quantitative, descriptive and transversal study evaluating the socio-demographic profile (12 items), health (15 items) and social conditions ( 4 items) of 58 elderly people aged 80 , through a structured questionnaire with 31 items. Results showed that sample comprised predominantly females $(68.96 \%)$, widows $(62.06 \%)$, aged $80-89$ years (79.31\%), with basic schooling (89.63\%) and retired (75.86\%). They owned their own house (65.51\%); they had children or grandchildren (89.65\%); they were Catholics (84.48\%); they had a health care plan (58.62\%); they featured eye problems (72.41\%) and employed corrective devices (72.41\%). Most had systemic arterial hypertension (55.17\%), they used hypotension drugs $(55.17 \%)$; they made regular visits to the doctor (63.79\%); underwent physiotherapy (53.44\%), practiced physical exercises (65.11\%) and classified their health status as fair or good (43.10\%). The social and demographic profile and the health and social conditions of eighty-year-old people may contribute
\end{abstract}


towards in-depth studies on this category. It is an issue of public health due to the growing population involved.

KEY WORDS: Elderly people aged 80 or more; Elderly; Longevity; Health profile; Social conditions.

\section{INTRODUÇÃO}

O Brasil apresentou considerável crescimento do número de idosos nos últimos anos passando de um contingente de $9,8 \%$ da população, em 2005, para 14,3\% da população, em 2015, fato este que deverá ter continuidade nas próximas décadas, segundo as projeções do Instituto Brasileiro de Geografia e Estatística (BRASIL, 2016A).

Os idosos com 80 anos ou mais de idade compõem o grupo que mais cresceu significativamente nos últimos tempos. Há vários termos para denominar estes indivíduos, como "idoso mais velho", "octogenário" ou "idoso longevo", este último como os define atualmente no meio científico. Este seleto grupo apresenta peculiaridades morfológicas, fisiológicas, psicológicas e socioeconômicas diferentes de idosos mais jovens, o que torna de interesse científico conhecer tais características dessa população. Entretanto, há riscos aos quais os octogenários estão expostos, pois a sociedade contemporânea não está preparada para receber a alta demanda desses indivíduos (MASTROENI et al., 2007; LENARDT et al., 2013).

Embora as alterações orgânicas, funcionais e psicológicas apresentem intensidade variada de acordo com a preparação prévia de cada indivíduo, o envelhecimento pode ser marcado pelo aumento das demandas sociais e econômicas, pois com ele cresce o índice de incapacidades e doenças crônicas não transmissíveis, responsáveis pela dependência funcional da população octogenária (NAHAS, 2008; NOGUEIRA et al., 2010; LENARDT et al., 2013).

Envelhecer com menores índices de doenças não transmissíveis depende de fatores não modificáveis, como sexo, idade e fatores genéticos, e fatores modificáveis, como alimentação, prática de atividade física, comportamento preventivo, estresse, vida social, entre outros, cujos mesmos são influenciados pelas condições socioeconômica, cultural e ambiental (BRASIL, 2010). Em virtude, das tendências expressivas para o aumento de idosos longevos com o decorrer do tempo, torna-se necessária a implementação de políticas públicas específicas para adequar os programas e as ações contribuintes para o envelhecimento saudável e com qualidade de vida (PORCIÚNCULA et al., 2014).

Diante do exposto, o presente estudo tem como objetivo caracterizar o perfil sociodemográfico, as condições de saúde e as condições sociais de idosos octogenários.

\section{METODOLOGIA}

O presente estudo é quantitativo, descritivo de corte transversal, cujo mesmo faz parte de um projeto que estimou avaliar 1.000 idosos, denominado "Caracterização das condições de saúde dos idosos do município de Passo Fundo/RS", aprovado pelo Comitê de Ética em Pesquisa em Seres Humanos da Universidade de Passo Fundo, sob o parecer n ${ }^{0} 447 / 2010$ e que está em consonância com a resolução 196/96 do Conselho Nacional de Saúde.

A população avaliada foi selecionada por uma amostragem aleatória simples e foi composta por 351 idosos. Como critérios de inclusão para o estudo foram selecionados indivíduos com idades a partir dos 80 anos, residentes da zona urbana do município de Passo Fundo/RS, com capacidade de comunicar-se verbalmente com o entrevistador e que concordaram em assinar o Termo de Consentimento Livre e Esclarecido, mediante prévia explicação e esclarecimento de dúvidas. Perante isso, foram excluídos do estudo os indivíduos que apresentassem alguma dificuldade que os impedissem de compreender e responder ao questionário, como acometimento auditivo ou disartria.

Os participantes responderam a um questionário estruturado que foi elaborado e adaptado pelos próprios pesquisadores, com base em outros estudos já referenciados na literatura sobre o perfil de idosos octogenários (PORCIÚNCULA et al., 2014; GRDEN et al., 2015; QUEIROZ et al., 2016). Diversos itens foram analisados compondo as variáveis caracterização do perfil 
sociodemográfico (gênero, faixa etária, escolaridade, ocupação laborativa, renda mensal individual, posse de casa própria, condição de moradia, estado civil, filhos, netos, tipo de religião e posse de plano de saúde-12 itens), condições de saúde (presença de alterações oculares, uso de dispositivos oculares para correção, autopercepção sobre a qualidade da visão, presença de tabagismo, presença de etilismo, presença de doenças associadas, tipos de doenças associadas, uso de medicamentos, tipos de medicamentos, consultas regulares ao médico, dificuldade nas atividades de vida diária, se já realizou fisioterapia, ocorrência de quedas, local de ocorrência da queda e autopercepção de saúde - 15 itens) e condições sociais (participação em cursos, participação em grupos de convivência, prática de atividade física e tipo de atividade física realizada -04 itens), totalizando 31 itens analisados pelos pesquisadores.

A coleta de dados foi realizada por um grupo de acadêmicos do curso de fisioterapia da Universidade de Passo Fundo, que foram previamente treinados para aplicar o questionário, no período de agosto de 2011 a setembro de 2012, nos seguintes locais: praças Tamandaré e Marechal Floriano, estabelecimentos públicos, estabelecimentos comerciais e Centro de Estudos sobre a Terceira Idade. O número de entrevistas realizadas nestes locais não foi homogêneo.

Ao fim da coleta de dados, as informações foram tabuladas no programa SPSS 18.0, construindo, assim, o banco de dados. Um matemático sem relação com o estudo foi requisitado para realizar a análise estatística descritiva dos dados, com o intuito de garantir a imparcialidade e máxima fidedignidade dos resultados.

\section{RESULTADOS}

Dos 351 indivíduos inicialmente recrutados que responderam ao questionário estruturado, apenas 58 foram identificados como octogenários $(16,52 \%$ da amostra inicial). Assim, os demais idosos, que não apresentaram mínima de 80 anos foram excluídos. Todos os idosos octogenários recrutados preencheram os critérios de inclusão do presente estudo e tiveram seus dados analisados. Desta forma, a Tabela 1 apresenta a caracterização do perfil sociodemográfico da amostra do presente estudo.

Tabela 1. Caracterização do perfil demográfico dos octogenários. Passo Fundo, RS, Brasil

(continua)

\begin{tabular}{lcc}
\hline & \multicolumn{2}{c}{ Representação } \\
\hline Variáveis & N & $\%$ \\
\hline Gênero & 40 & 68,96 \\
\hline Feminino & 18 & 31,03 \\
Masculino & & \\
\hline Faixa etária & 46 & 79,31 \\
\hline $80-89$ anos & 08 & 13,79 \\
$90-99$ anos & 04 & 6,89 \\
$\geq 100$ anos & & \\
\hline Escolaridade & 32 & 55,17 \\
\hline Ensino fundamental incompleto & 09 & 15,51 \\
Ensino fundamental completo & 02 & 3,44 \\
Ensino médio incompleto & 09 & 15,51 \\
Ensino médio completo & - & - \\
Ensino superior incompleto & 06 & 10,34 \\
Ensino superior completo & & \\
\hline Ocupação laborativa & & \\
\hline
\end{tabular}


(conclusão)

\begin{tabular}{|c|c|c|}
\hline \multirow[b]{2}{*}{ Variáveis } & \multicolumn{2}{|c|}{ Representação } \\
\hline & $\mathbf{N}$ & $\%$ \\
\hline Aposentado (a) & 44 & 75,86 \\
\hline Pensionista & 04 & 6,89 \\
\hline Nunca trabalhou & 08 & 13,79 \\
\hline Do lar & 02 & 3,44 \\
\hline \multicolumn{3}{|l|}{ Renda mensal individual } \\
\hline Até 1 salário mínimo & 27 & 46,55 \\
\hline 2 salários mínimos & 13 & 22,41 \\
\hline 3 salários mínimos & 11 & 18,96 \\
\hline 4 salários mínimos ou mais & 07 & 12,06 \\
\hline \multicolumn{3}{|l|}{ Casa própria } \\
\hline Sim & 38 & 65,51 \\
\hline Não & 20 & 34,48 \\
\hline \multicolumn{3}{|l|}{ Condição de moradia } \\
\hline Só & 30 & 51,72 \\
\hline Acompanhado & 28 & 48,27 \\
\hline \multicolumn{3}{|l|}{ Estado civil } \\
\hline Solteiro (a) & 06 & 10,34 \\
\hline Casado (a) & 14 & 24,13 \\
\hline Viúvo (a) & 36 & 62,06 \\
\hline Divorciado (a) / Separado (a) & 02 & 3,44 \\
\hline \multicolumn{3}{|l|}{ Filhos } \\
\hline $\operatorname{Sim}$ & 52 & 89,65 \\
\hline Não & 06 & 10,34 \\
\hline \multicolumn{3}{|l|}{ Netos } \\
\hline Sim & 52 & 89,65 \\
\hline Não & 06 & 10,34 \\
\hline \multicolumn{3}{|l|}{ Religião } \\
\hline Catolicismo & 49 & 84,48 \\
\hline Evangelismo & 05 & 8,62 \\
\hline Protestantismo & 01 & 1,72 \\
\hline Espiritismo & 01 & 1,72 \\
\hline Outra & 02 & 3,44 \\
\hline \multicolumn{3}{|l|}{ Possui plano de saúde } \\
\hline Sim & 34 & 58,62 \\
\hline Não & 24 & 41,37 \\
\hline
\end{tabular}

Legenda: $\mathrm{N}=$ valor absoluto; $\%=$ valor relativo

Fonte: Dados da pesquisa 
Ao analisar as características demográficas da amostra estudada, observou-se que a maioria pertencia ao gênero feminino $(68,96 \%)$, concentrava-se na faixa etária de 80 a 89 anos $(79,31 \%)$ e $55,17 \%$ apresentavam baixo nível de escolaridade (ensino fundamental incompleto). A maioria dos idosos encontrava-se aposentada $(75,86 \%)$ e com renda de até um salário mínimo (46,55\%). Os indivíduos com casa própria somaram $65,51 \%$ e, ainda, a maioria relatou morar sozinho (51,72\%). A condição de viuvez foi relatada por $62,06 \%$ dos indivíduos e $89,65 \%$ da amostragem relatou possuir filhos e netos. Segundo a religiosidade, a maioria da amostra relatou pertencer à religião católica $(84,48 \%)$. E, por fim, $58,62 \%$ dos indivíduos relataram fazer uso de planos de saúde.

A Tabela 2 apresenta a caracterização das condições de saúde da amostra do presente estudo.

Tabela 2. Caracterização das condições de saúde dos octogenários. Passo Fundo, RS, Brasil

(continua)

\begin{tabular}{|c|c|c|}
\hline \multirow[b]{2}{*}{ Variáveis } & \multicolumn{2}{|c|}{ Representação } \\
\hline & $\mathbf{N}$ & $\%$ \\
\hline \multicolumn{3}{|l|}{ Alterações oculares } \\
\hline Sim & 42 & 72,41 \\
\hline Não & 16 & 27,58 \\
\hline \multicolumn{3}{|c|}{ Uso de dispositivos para correção } \\
\hline Sim & 42 & 72,41 \\
\hline Não & 16 & 27,58 \\
\hline \multicolumn{3}{|c|}{ Autopercepção sobre a qualidade da visão } \\
\hline Sim & 11 & 18,96 \\
\hline Não & 30 & 51,72 \\
\hline \multicolumn{3}{|l|}{ Tabagismo } \\
\hline Sim & 13 & 22,41 \\
\hline Não & 45 & 77,58 \\
\hline \multicolumn{3}{|l|}{ Etilismo } \\
\hline Sim & 08 & 13,79 \\
\hline Não & 50 & 86,20 \\
\hline \multicolumn{3}{|l|}{ Doenças associadas } \\
\hline Sim & 46 & 79,31 \\
\hline Não & 12 & 20,68 \\
\hline \multicolumn{3}{|l|}{ Tipos de doenças associadas } \\
\hline Diabetes & 10 & 17,24 \\
\hline Dislipidemia & 12 & 20,68 \\
\hline Hipertensão arterial sistêmica & 32 & 55,17 \\
\hline Doença cardiovascular & 12 & 20,68 \\
\hline Osteoporose & 16 & 27,58 \\
\hline Osteoartrose & 16 & 27,58 \\
\hline Acidente vascular encefálico & 01 & 1,72 \\
\hline Neoplasia & 01 & 1,72 \\
\hline Uso de medicamentos & & \\
\hline
\end{tabular}


(conclusão)

\begin{tabular}{|c|c|c|}
\hline \multirow[b]{2}{*}{ Variáveis } & \multicolumn{2}{|c|}{ Representação } \\
\hline & $\mathbf{N}$ & $\%$ \\
\hline Sim & 39 & 67,24 \\
\hline Não & 19 & 32,75 \\
\hline \multicolumn{3}{|l|}{ Tipos de medicamentos } \\
\hline Diurético & 19 & 32,75 \\
\hline Hipotensores & 32 & 55,17 \\
\hline Antidepressivos & 10 & 17,24 \\
\hline Psicoativos & 07 & 12,06 \\
\hline Outros & 22 & 37,93 \\
\hline \multicolumn{3}{|c|}{ Consultas regulares ao médico } \\
\hline Sim & 37 & 63,79 \\
\hline Não & 15 & 25,86 \\
\hline Apenas quando adoece & 06 & 10,34 \\
\hline \multicolumn{3}{|c|}{ Dificuldade nas atividades de vida diária } \\
\hline Sim & 16 & 27,58 \\
\hline Não & 42 & 72,41 \\
\hline \multicolumn{3}{|l|}{ Já fez fisioterapia? } \\
\hline Sim & 31 & 53,44 \\
\hline Não & 27 & 46,55 \\
\hline \multicolumn{3}{|c|}{ Ocorrência de queda nos últimos 06 meses } \\
\hline Sim & 15 & 25,86 \\
\hline Não & 43 & 74,13 \\
\hline \multicolumn{3}{|c|}{ Local onde ocorreu a queda } \\
\hline Em casa & 09 & 15,51 \\
\hline Na rua & 05 & 8,62 \\
\hline \multicolumn{3}{|l|}{ Autopercepção de saúde } \\
\hline Péssima & - & - \\
\hline Ruim & 04 & 6,89 \\
\hline Regular & 25 & 43,10 \\
\hline Boa & 25 & 43,10 \\
\hline Ótima & 04 & 6,89 \\
\hline
\end{tabular}

Legenda: $\mathrm{N}=$ valor absoluto; $\%=$ valor relativo

Fonte: Dados da pesquisa 
Em relação às condições de saúde dos idosos longevos, $72,41 \%$ da amostra apresentaram alterações oculares e a mesma porcentagem fazia uso de dispositivos para correção, portanto $51,72 \%$ dos indivíduos classificaram a visão como regular. Em relação ao uso de drogas lícitas como o tabaco e o álcool, a grande parte dos estudados negou ser usuária $(77,58 \%$ e 86,20\%, respectivamente). A hipertensão arterial sistêmica foi a doença associada mais prevalente $(55,17 \%)$, sendo que $67,24 \%$ da amostra faziam uso de medicamentos, principalmente de hipotensores $(55,17 \%)$. Em relação às consultas médicas, $63,79 \%$ dos indivíduos as realizavam frequentemente. A maioria da amostra demonstrou realizar as atividades de vida diária normalmente $(72,41 \%)$ e $53,44 \%$ confirmaram já ter utilizado o serviço de fisioterapia alguma vez na vida. A maioria dos indivíduos não relatou a ocorrência de quedas nos últimos seis meses $(74,13 \%)$ e os que relataram o evento ocorreu em casa (15,51\%). Em relação à autopercepção de saúde, 43,10\% dos indivíduos relataram sua saúde como regular e a mesma porcentagem determinou sua saúde como boa.

Na Tabela 3 estão apresentados os dados referentes às condições sociais da amostra estudada.

Observou-se que, em relação às condições sociais dos indivíduos, a maioria não realizava qualquer curso e nem frequentava grupos de terceira idade (84,48\% e 58,62\%, respectivamente), mas possuíam o hábito de praticar atividade física (65,51\%), em que o tipo de atividade que prevaleceu foi o alongamento (46,55\%).

Tabela 3. Caracterização das condições sociais dos octogenários. Passo Fundo, RS, Brasil

\begin{tabular}{|c|c|c|}
\hline \multirow[b]{2}{*}{ Variáveis } & \multicolumn{2}{|c|}{ Representação } \\
\hline & $\mathbf{N}$ & $\%$ \\
\hline \multicolumn{3}{|c|}{ Participação em cursos } \\
\hline Sim & 09 & 15,51 \\
\hline Não & 49 & 84,48 \\
\hline \multicolumn{3}{|c|}{ Participação em grupos de convivência } \\
\hline Sim & 24 & 41,37 \\
\hline Não & 34 & 58,62 \\
\hline \multicolumn{3}{|c|}{ Prática de atividade física } \\
\hline Sim & 38 & 65,51 \\
\hline Não & 20 & 34,48 \\
\hline \multicolumn{3}{|c|}{ Tipo de atividade física } \\
\hline Caminhada & 11 & 18,96 \\
\hline Alongamento & 27 & 46,55 \\
\hline Dança & 09 & 15,51 \\
\hline Hidroginástica & 04 & 6,89 \\
\hline Musculação & 01 & 1,72 \\
\hline Outro & 07 & 12,06 \\
\hline
\end{tabular}

Legenda: $\mathrm{N}=$ valor absoluto; \% = valor relativo

Fonte: Dados da pesquisa 


\section{DISCUSSÃO}

A população idosa brasileira apresentou aumento considerável nas últimas décadas, em virtude da articulação e transição demográfica e epidemiológica no país, especialmente o grupo os indivíduos com 80 anos ou mais. Neste sentido, a reorganização dos sistemas de saúde e previdenciário do Estado e da sociedade se faz necessária (PORCIÚNCULA et al., 2014). O que torna de interesse científico o conhecimento acerca do perfil e das condições de saúde e sociais dos idosos octogenários.

O perfil sociodemográfico explanado na presente pesquisa corrobora os achados de outros estudos que tratam da saúde dos idosos acima dos 80 anos. Neste estudo, podemos observar que há o predomínio do gênero feminino, evidenciando maior longevidade das mulheres em relação aos homens. Esta informação é notada nos estudos de Lenardt e Carneiro (2013), Grden et al. (2015) e Queiroz et al. (2016) que observaram em seu estudo a prevalência de mulheres viúvas. Este fator reflete os hábitos gerais de cada sexo, em que a mulher geralmente está menos exposta a fatores de risco de mortalidade, ao tabagismo e etilismo, além de estar mais inserida em ações de prevenção e autocuidado, pois recorrem mais aos serviços de saúde (MOURA; DOMINGOS; RASSY, 2010).

Dados governamentais apontam que, desde os anos de 1940, houve aumento de 30 anos na expectativa de vida dos brasileiros, passando de uma média de 45,5 anos para uma média de 75,5 anos (BRASIL, 2016B). Além disso, pode-se observar que a maioria dos indivíduos que chegam à terceira década de vida, sobretudo os octogenários, é mulhere concentrada na faixa etária entre os 80-89 anos (PORCIÚNCULA et al., 2014). O que concorda com o presente estudo, cujo mesmo observou predominância dos indivíduos nesta faixa etária e do gênero feminino. Talvez, a expectativa de vida dos brasileiros possa ter sido um fator que tenha contribuído para tais achados.

No Brasil, a proporção de idosos mais velhos que vivem sozinhos é maior do que a de idosos mais jovens (CAMARANO, 2002), o que deve ser levado em consideração na caracterização do perfil de idosos octogenários, haja vista que a dependência funcional e os fatores de risco aumentam com a idade, especialmente acima da faixa etária dos 80 anos. Neste sentido, Rosset et al. (2011) caracterizaram o perfil socioeconômico de idosos octogenários residentes nos municípios de Ribeirão Preto/SP e Caxias do Sul/RS, onde se observou que a maioria relatou possuir casa própria $(64,8 \%)$ e um número expressivo destes residia sozinho, sendo 13,2\% em Ribeirão Preto e 26,7\% em Caxias do Sul. Tais informações corroboram com os resultados obtidos no presente estudo, visto que $65,51 \%$ da amostra relataram ser proprietária de sua própria moradia e um número muito superior à literatura relatou residir sozinho em seus lares $(51,72 \%)$.

Observou-se neste estudo que os octogenários apresentaram predominantemente escolaridade básica, sendo que $55,17 \%$ nem chegou a concluir o ensino fundamental, o que também foi observado no estudo realizado por Porciúncula et al. (2014), cujo mesmo observou que a maioria dos longevos não tinha qualquer tipo de escolaridade ou tinha escolaridade básica. Isso pode ser atribuído ao fato de que a população acima dos 80 anos tenha vivido em um período escolar em que não havia a abrangência em massa do ensino público. Apenas e recentemente o Brasil adotou políticas e ações públicas na tentativa de modificar este quadro, logo se espera encontrar um nível baixo de escolaridade entre os idosos octogenários (PORCIÚNCULA et al., 2014).

Grden et al. (2015) realizaram um estudo epidemiológico e transversal, que objetivou identificar as características sociodemográficas e de acesso de octogenários aos serviços de saúde. Dos 56 idosos longevos cadastrados em 13 centros de convivência do idoso no município de Ponta Grossa/PR que participaram da pesquisa, observou-se que a maior parte da amostra possuía ensino fundamental incompleto $(71,4 \%)$, com renda individual igual ou menor a dois salários mínimos $(89,3 \%)$ e moravam sozinhos $(46,4 \%)$. Sendo essas variáveis compatíveis com o atual estudo.

Ter uma boa condição econômica é de suma importância para ter acesso aos serviços de saúde de boa qualidade. Sabendo-se que é reduzida a possibilidade de longevos conseguir um emprego, o que restringe ao indivíduo idoso apenas sobreviver com sua aposentadoria ou pensão como fonte de renda, como observado em um 
estudo de caracterização de idosos, em que a maior parte da amostra relatou possuir baixa renda (LENARDT et al., 2013). Evidenciando estes apontamentos, a maioria dos indivíduos deste estudo relatou ser composta por indivíduos aposentados $(75,86 \%)$, o que concorda com Porciúncula et al. (2014), cujo mesmo identificou que a maioria dos octogenários era composta por aposentados e tinha a aposentadoria como principal fonte de renda (43,9\%).

Com relação ao cônjuge, um estudo identificou que os homens longevos são predominantemente casados e residem com seus pares, enquanto as mulheres longevas são predominantemente viúvas e residem com seus filhos ou netos (PORCIÚNCULA et al., 2014). Apesar de uma ligeira maioria $(51,72 \%)$ dos octogenários terem relatado morar sozinhos, neste estudo observou-se que, no geral, a predominância do gênero feminino pode ter sido um fator capaz de influenciar os resultados, caracterizando a amostra em uma condição de viuvez e com filhos e/ou netos.

Os estudos têm explorado as implicações da religiosidade sobre as respostas físicas e mentais de indivíduos (DUARTE et al., 2008). Assim, na mesma proporção em que os indivíduos envelhecem há aumento da busca por aspectos espirituais, sendo que esse tipo de atividade é mais comum na terceira idade do que em qualquer outra faixa etária (GOLDESTEIN et al., 1993). Com relação à religiosidade, observou-se no presente estudo a predominância do catolicismo $(84,48 \%)$, o que concorda com os achados de Duarte et al. (2008), em que se observou que os idosos octogenários eram predominantemente católicos (75,3\%), seguidos pelas religiões evangélica (13,9\%), espírita $(4,4 \%)$ e outras, assim como identificado na presente amostra.

Os idosos que residem em áreas urbanas de municípios de médio ou grande porte podem apresentar maiores chances de possuir melhores valores da sua renda, o que poderia favorecer a utilização de planos de saúde (ROSSET et al., 2011). Segundo Rosset et al. (2011), em seu estudo, grande parte dos octogenários fazem uso de plano de saúde privado (66,7\%), em relação a uma parcela menor que faz uso do Sistema Único de Saúde $(33,3 \%)$, o que vai ao encontro do presente estudo em que se observou que 58,62\% da amostra relataram possuir plano de saúde particular, sendo que eram residentes do município de Passo Fundo/RS, uma cidade de grande porte com 197 mil habitantes contabilizados no ano de 2016. Conhecer tais achados pode servir de subsídios para compreender a influência que as variáveis analisadas neste estudo podem exercer sobre a utilização do plano de saúde privado, o acesso de saúde e a qualidade de vida dos idosos octogenários.

As alterações visuais são tidas como fatores de risco para a dependência funcional dos octogenários, sendo necessário conhecer tais fatores (RIBEIRO et al., 2015). Este problema pode ser um agravante capaz de produzir, além da dependência, situações de constrangimento para iniciar e manter conversas, dificuldade para sair de casa, isolamento social, perda de papéis ocupacionais, limitações de mobilidade e condições de fragilidade (VEIGA et al., 2016). Embora haja escassez na literatura sobre as condições visuais dos idosos octogenários, tal variável foi levada em consideração neste estudo, em que se observou que $72,41 \%$ da amostra relataram apresentar alterações oculares e fazer uso de dispositivo para correção, sendo que a maioria classificou sua visão como regular. Estes achados corroboram com o estudo desenvolvido por Menezes e Bachion (2012) em que observaram que $81,4 \%$ da amostra apresentaram alterações visuais, porém apenas 44,1\% faziam uso de dispositivos para correção. Embora não tenham sido avaliados exclusivamente idosos octogenários, a média de idade foi de 78,4 47,8 anos, próxima à condição de longevidade.

Por vezes, os idosos podem estar vulneráveis ao tabagismo e ao etilismo, pois apresentam diversificados problemas de saúde e fazem uso de medicamentos, que associados a tais substâncias nocivas, tornam-nos suscetíveis ao agravamento do seu quadro clínico e à dificuldade de recuperação e interação social. Esta condição tende a se agravar futuramente, pois há aumento da população idosa (SENGE et al., 2011). Levando em consideração a vulnerabilidade ao tabagismo e etilismo a qual os idosos estão sujeitos, é que o presente estudo analisou estas variáveis, e os resultados aqui obtidos corroboram com os achados do estudo de Zaitune et al. (2012) em que se identificou que $12,2 \%$ da população idosa ( $6 \%$ entre os idosos longevos) eram tabagistas, 
principalmente entre os homens, e com os achados do estudo de Lin et al. (2011) com idosos acima dos 65 anos que identificaram a prevalência do uso de álcool em $16,1 \%$ da população geriátrica durante a sua vida e de $1,5 \%$ no ano anterior à pesquisa.

De acordo com Mendes, Moraes e Gomes (2014), a maior parte dos longevos refere possuir doenças relacionadas ao sistema circulatório (78\%) e que o menor nível de escolaridade pode ser um fator determinante para tal condição. Com base nisto, Zanin et al. (2017) evidenciaram que dos 351 idosos avaliados no município de Passo Fundo/RS, 198 (56,41\%) apresentavam a hipertensão arterial sistêmica como comorbidade, porém apenas $1,5 \%$ desta população era longeva. Estas informações confirmam os dados presentes neste estudo, pois a maior parte da amostra relatou possuir como doença associada à hipertensão arterial sistêmica e possuía baixo nível de escolaridade.

Com o aumento da idade ocorre também o aumento do consumo de medicamentos. O estudo de Brito et al. (2014) demonstrou que o uso de medicamentos está estatisticamente relacionado ao aumento da dependência funcional entre os idosos longevos, fazendo-se necessário identificar nas condições de saúde desta população o uso de medicamentos e sua finalidade. Baseado nisto, observou-se que neste estudo a maioria dos idosos fazia uso de medicamentos (67,24\%), especialmente de hipotensores $(55,17 \%)$, achados estes que corroboram com o estudo de Neves et al. (2013), em que o mesmo observou que a prevalência do uso de medicamentos em $85,5 \%$ da população idosa, sendo a maioria com indicação médica para o sistema cardiovascular $(42,9 \%)$.

Torna-se de grande valia compreender a frequência que os idosos octogenários frequentam aos serviços médicos e de fisioterapia, o que pode ser um indicador de saúde desta população. Em um estudo realizado por Nogueira et al. (2010), observou-se que dos idosos longevos frequentava regularmente os serviços de saúde. Neste estudo, a maioria dos indivíduos relatou fazer consultas regulares ao médico $(63,79 \%)$ ou já ter recorrido aos serviços de fisioterapia $(53,44 \%)$ alguma vez na vida. Somado a isso, a maioria dos longevos entrevistados relatou fazer atividade física $(65,51 \%)$.
A incapacidade funcional é um fator determinante na vida diária do idoso longevo. É atribuída predominantemente ao gênero feminino, pois as mulheres podem apresentar maior predisposição ao desenvolvimento de complicações físicas e mentais (NOGUEIRA et al., 2009). Estudos recentes apresentam dados quanto à autonomia física da população geriátrica. Um estudo de Recife/PB demonstrou que os octogenários têm boa autonomia e independência na realização das suas atividades de vida diária (PORCIÚNCULA et al., 2014). Outro estudo realizado por Brito et al. (2010) demonstrou que a $80,4 \%$ dos longevos relatou ser independente para suas atividades de vida diária. O que concorda com o presente estudo visto que $72,41 \%$ dos entrevistados relataram ser funcionalmente independente.

A ocorrência de quedas na população geriátrica é uma das preocupações mais frequentes no sistema de saúde pelas consequências que estas produzem à qualidade de vida destes indivíduos (KUMMER; FAREZIN; WIBELINGER, 2014). Brito et al. (2010) e Brito et al. (2014) observaram que entre os longevos há baixo índice de quedas $(27,7 \%)$ e que a ocorrência deste evento associou-se estatisticamente ao gênero feminino, à condição de não ter um companheiro (a), à não realização de atividade física, à presença de doenças crônicas e à dependência funcional. Neste estudo também se observou baixa ocorrência de quedas entre os idosos longevos $(25,86 \%)$ e alguns aspectos concordam com os autores supracitados, haja vista que a maioria da amostra relata fazer atividade física e ser funcionalmente independente. Contrapartida observou-se que a maioria da amostra era do gênero feminino, não tinham cônjuge (a maioria era solteira, viúva ou divorciada) e apresentavam alto índice de doenças associadas.

O idoso com vida social ativa consegue retardar sua perda de memória, além de diminuir as chances de desenvolver doenças psicológicas (NOGUEIRA et al., 2009). Todavia, pesquisas nacionais revelaram que a maioria dos idosos não participa de atividades sociais e atribuem isto à presença de doenças, incapacidades cognitivas e funcionais, dores crônicas, dificuldade de acesso, baixa escolaridade e falta de recursos financeiros (SILVA; THER, 2000; VAROTO; TRUZZI; PAVARINI, 2004; CELICH; GALON, 2009). 
Entre os longevos há baixa prevalência daqueles que participam de grupos de convivência, como no estudo desenvolvido por Kummer, Farezin e Wibelinger (2014), em Passo Fundo/RS, em que se observou que dos 126 idosos participantes de grupos de convivência, os longevos foram o grupo de menor frequência $(3,2 \%)$. Confirmando isto, um estudo desenvolvido na Alemanha com 2.300 idosos apontou que a baixa escolaridade, as piores condições de saúde e a idade avançada são fatores que restringem a participação dos idosos em grupos de atividades sociais (KRUPPER; ZICK, 2011). Tais estudos corroboram com a presente pesquisa, visto que a minoria dos octogenários relatou ser participante de grupos de convivência e apresentava predominantemente baixa escolaridade e alto índice de doenças associadas.

Além disso, os octogenários são mais seletivos. Priorizam os vínculos afetivos já estabelecidos e não se envolvem em muitas e novas relações pessoais, mantendo as relações afetivas construídas ao longo da vida, principalmente com os familiares (CHARLES; CARSTENSEN, 2010). Todavia, os grupos de convivência podem ajudar a prevenir agravos, promover a saúde e proporcionar melhor qualidade de vida para os idosos (KUMMER; FAREZIN; WIBELINGER, 2014).

A prática de atividade física pode ser um fator influente na capacidade funcional dos longevos (RIBEIRO et al., 2015). De acordo com o estudo desenvolvido por Krug et al. (2016), a prevalência de atividade física entre os octogenários foi de $19 \%$, sendo que os fatores idade avançada, pior percepção de saúde e diagnóstico de hipertensão arterial sistêmica contribuíram para o baixo índice de atividade física nesta população. Indo de encontro aos achados descritos, pois os resultados deste estudo apontam para um elevado índice de longevos praticantes de atividade física $(65,51 \%)$, sendo que a prática do alongamento foi a mais relatada (46,55\%), embora a maioria da amostra tenha referido ser diagnosticada com hipertensão arterial sistêmica.

O relato de uma autopercepção de saúde pode se relacionar ao nível de capacidade funcional entre os idosos longevos, sendo que quanto melhor a autopercepção de saúde referida melhor é a capacidade funcional desta população (NOGUEIRA et al., 2010). Ao observar os dados sobre as percepções de saúde, evidencia-se que a maior parte dos idosos octogenários vê sua saúde como regular e boa. Enquanto Queiroz et al. (2016) e Nogueira et al. (2010) mostram que em sua pesquisa a maior parte dos idosos percebia sua saúde como boa ou ótima.

Tais achados também foram encontrados em um estudo realizado com octogenários de Recife/PB, em que se revelou que a grande maioria $(71,1 \%$ dos homens e $62,8 \%$ das mulheres) referia-se à sua saúde como boa ou ótima (PORCIÚNCULA et al., 2014), concordando novamente com o presente estudo, visto que a maioria da amostra revelou sua saúde como boa $(43,10 \%)$.

O presente estudo apresenta algumas limitações. Alguns itens abordados neste estudo foram pouco explanados na literatura até o presente momento, sobretudo em populações octogenárias como, por exemplo, a frequência de utilização dos serviços de saúde, o uso de medicamentos ou as condições visuais e oculares dos idosos, o que dificultou a discussão deste estudo. Outro quesito limitante pode ter sido o modo de escolha de seleção da amostra, o que pode ter contribuído para o pequeno número de idosos longevos recrutados neste estudo. Apesar disto, não foi inviável a geração de dados para a fundamentação deste estudo. Assim, sugere-se a possibilidade de investigações futuras sobre a caracterização do perfil sociodemográfico, das condições de saúde e das condições sociais de idosos octogenários.

\section{CONCLUSÃO}

O perfil sociodemográfico, as condições de saúde e as condições sociais dos idosos descritas neste estudo podem contribuir para o conhecimento sobre os octogenários, o que torna isto uma questão de saúde pública em virtude do progressivo crescimento populacional destes indivíduos. 


\section{REFERÊNCIAS}

BRASIL. Em 10 anos, cresce número de idosos no Brasil. Portal Brasil, 2016A. Disponível em: <http://www. brasil.gov.br/economia-e-emprego/2016/12/em-10-anoscresce-numero-de-idosos-no-brasil $>$. Acesso em: 28 de maio de 2017.

BRASIL. Expectativa de vida no Brasil sobe para 75,5 anos em 2015. Portal Brasil, 2016B. Disponível em: < http:// www.brasil.gov.br/governo/2016/12/expectativa-de-vidano-brasil-sobe-para-75-5-anos-em-2015> . Acesso em: 16 de maio de 2017.

BRASIL. Ministério da Saúde. Secretaria de Atenção à Saúde, Departamento de Ações Programáticas e Estratégicas. Área Técnica Saúde do Idoso. Atenção à saúde da pessoa idosa e envelhecimento. Brasília, DF, 2010, 44p.

BRITOT.A. et al. Capacidade funcional e fatores associados em idosos longevos residentes em comunidade: estudo populacional no Nordeste do Brasil. Fisioter Pesq, v. 21, n. 4, p. 308-313, 2014.

BRITO T. A. et al. Quedas e capacidade funcional em idosos longevos residentes em comunidade. Texto Contexto Enferm, v. 22, n. 1, p. 43-51, 2013.

CAMARANO, A. A. Envelhecimento da população brasileira: uma contribuição demográfica. Rio de Janeiro: Instituto de Pesquisa Econômica Aplicada; 2002.

CELICH, K.L.S.; GALON, C. Dor crônica em idosos e sua influência nas atividades da vida diária e convivência social. Rev Bras Geriatr Gerontol, v. 12, n. 3, p. 345 359, 2009.

CHARLES, S.T.; CARSTENSEN, L. L. Social and Emotional Aging. Annu Rev Psychol, v.61, n.1, p. 383-409, 2010.

DUARTE, Y. A. O. et al. Religiosidade e envelhecimento: uma análise do perfil de idosos do município de São Paulo. Saúde Coletiva, v. 5, n. 24, p. 173-177, 2008.

GOLDESTEIN, L. L. Desenvolvimento do adulto e da religiosidade: uma questão de fé. In: NÉRI, A. L. (Org.). Qualidade de vida e idade madura. Campinas: Papirus,
1993. p. 83-108.

GRDEN, C. R. B. et al. Características sociodemográficas e de acesso de longevos aos serviços de saúde. Cienc Cuid Saude, v. 14, n. 4, p. 1505-1512, 2015.

KRUG R. R. et al. Prevalence of commuting physical activity and associated factors in long-lived older adults. Rev Bras Cineantropom Desempenho Hum, v. 18, n. 5, p. 520-529, 2016.

KUMMER, J. A.; FAREZIN, S.; WIBELINGER, L. M. Caracterização das condições de saúde de idosos participantes de grupos de convivência. EFDeportes, v. 19, n. 194, p. 1, 2014.

KÜPPER. B.; ZICK, A. Inverse gender gap in Germany: social dominance orientation among men and women. Int J Psychol, v. 46, n.1, p.33-45, 2011.

LENARDT, M. H. et al. Prevalence of pre-frailty for the component of gait speed in older adults. Rev Latino-Am Enfermagem, v. 21, n. 3, p. 734-774, 2013.

LENARDT, M. H.; CARNEIRO, N. H. K. Associação entre as características sociodemográficas e a capacidade funcional de idosos longevos da comunidade. Cogitare Enferm, v. 18, n. 1, p. 13-20, 2013.

LIN, J.C. et al. Alcohol, tobacco, and nonmedical drug use disorders in U.S. Adults aged 65 years and older: data from the 2001-2002 National Epidemiologic Survey of Alcohol and Related Conditions. Am J Geriatr Psychiatry, v.19, n.3, p.292-299, 2011.

MASTROENI, M. F. et al. Perfil demográfico de idosos da cidade de Joinville, Santa Catarina: Estudo de base domiciliar. Rev Bras Epidemiol, v. 10, n. 2, p. 190-201, 2007.

MENDES, G. S.; MORAES, C. F.; GOMES, L. Prevalência de hipertensão arterial sistêmica em idosos no Brasil entre 2006 e 2010. Rev Bras Med Fam Comunidade, v. 9, n. 32, p. 273-278, 2014.

MENEZES R. L.; BACHION, M. M. Condições visuais autorrelatadas e quedas em idosos institucionalizados. Rev Bras Oftalmol, v. 71, n. 1, p. 23-27, 2012. 
MOURA, M. A. V.; DOMINGOS, A. M.; RASSY, M. E. C. A qualidade na atenção à saúde da mulher idosa: um relato de experiência. Esc Anna Nery, v. 14, n. 4, p. 848-855, 2010

NAHAS, M. V. Atividade física, saúde e qualidade de vida. 4. ed. Londrina: Midiograf, 2008.

NEVES, S. J. F. et al. Epidemiologia do uso de medicamentos entre idosos em área urbana do Nordeste do Brasil. Rev Saúde Pública, v. 47, n. 4, p. 759-768, 2013.

NOGUEIRA, S. L. et al. Fatores determinantes da capacidade funcional em idosos longevos. Rev Bras Fisioter, v. 14, n. 4, p. 322-329, 2010.

PORCIÚNCULA, R. C. R. et al. Perfil socioepidemiológico e autonomia de longevos em Recife-PE, Nordeste do Brasil. Rev Bras Geriatr Gerontol, v. 17, n. 2, p. 315325, 2014.

QUEIROZ, D. B. B. et al. Funcionalidade, aptidão motora e condições de saúde em idosos longevos residentes em domicílio. Arq Ciênc Saúde, v. 23, n. 2, p. 47-53, 2016.

RIBEIRO, D. K. M. N. et al. Fatores contributivos para a independência funcional de idosos longevos. Rev Esc Enferm USP, v. 49, n. 1, p. 89-95, 2015.

ROSSET, I. et al. Diferenciais socioeconômicos e de saúde entre duas comunidades de idosos longevos. Rev Saúde Pública, v. 45, n. 2, p. 391-400, 2011.

SENGER, A. E. V. et al. Alcoolismo e tabagismo em idosos: relação com ingestão alimentar e aspectos socioeconômicos. Rev Bras Geriatr Gerontol, v. 14, n. 4, p. 713-9, 2011.

SILVA, I.R.; GUNTHER, I.A. Papéis sociais e envelhecimento em uma perspectiva de curso de vida. Psc: Teoria e Pesquisa, v.16, n.1, p. 31-40, 2000.

VAROTO, V.A.G.; TRUZZI, O.M.S.; PAVARINI, S.C.L. Programas para idosos independentes: um estudo sobre seus egressos e a prevalência de doenças crônicas. Texto e Contexto Enferm, v.13, n. 1, p. 107-114, 2004.

VEIGA, B. et al. Avaliação de funcionalidade e incapacidade de idosos longevos em acompanhamento ambulatorial utilizando a WHODAS 2.0. Rev Bras Geriatr Gerontol, v. 19, n. 6, p. 1015-1021, 2016.

ZAITUNE, M. P. A. et al. Fatores associados ao tabagismo em idosos: Inquérito de Saúde no Estado de São Paulo (ISA-SP). Cad Saúde Pública, v. 28, n. 3, p. 583-595, 2012

ZANIN, C. et al. Autopercepção de saúde em idoso com hipertensão arterial sistêmica. Rev Interdisciplinar de Ciências Médicas - MG, v. 1, n.1, p. 26-33, 2017.

Recebido em: Aceito em: 30 de maio de 2017 